\title{
All hadronic B decay trigger with the CDF Silicon Vertex Tracker
}

\author{
S. Donati \\ INFN Sezione di Pisa, Via Livornese, 1291, 56010 San Piero a Grado, Pisa, Italy \\ for the CDF Collaboration, \\ Email: 'donati@pi.infn.it'
}

ABSTRACT: Silicon Vertex tracks are of fundamental importance for reconstructing B meson decays at a hadron collider. The upgraded CDF detector will deploy an online Silicon Vertex Tracker in the level 2 trigger. We have studied how this new device exploits the Tevatron large B meson production to select hadronic $\mathrm{B}$ decays fundamental for measuring $\mathrm{CP}$ violation and $\mathrm{B}_{s}$ mixing.

\section{Introduction}

The Tevatron $p \bar{p}$ Collider and the CDF detector are presently being upgraded for run II operation (to begin in the year 2000) [핌]. The Silicon Vertex Tracker (SVT) has been designed to reconstruct silicon vertex tracks in the transverse plane to the beam axis at the level 2 of CDF trigger [īi]. The SVT receives input tracks reconstructed in the central drift chamber (COT) by the eXtremely Fast Tracker (XFT) at level 1 , and the digitized pulse heights from the silicon vertex detector (SVX) front end electronics. The SVT determines the transverse momentum $\left(p_{t}\right)$, the azimuthal angle $(\phi)$ and the impact parameter $(d)$ of all the tracks with $p_{t}>2 \mathrm{GeV} / \mathrm{c}$. Tracking quality is very close to offline: $\sigma_{p t}=$ $0.003 \cdot p_{t}^{2} \mathrm{GeV} / \mathrm{c}\left(p_{t}\right.$ in $\left.\mathrm{GeV} / \mathrm{c}\right), \sigma_{\phi}=1 \mathrm{mrad}$ and $\sigma_{d}=35 \mu \mathrm{m}\left(\right.$ at $\left.p_{t}=2 \mathrm{GeV} / \mathrm{c}\right)$. Precision measurement of the track impact parameter at the trigger level permits triggering directly on secondary vertices. This exploits the Tevatron large $b \bar{b}$ cross section much more efficiently than leptonic triggers. In this paper we report on the simulation of a trigger which uses the SVT to select fully hadronic B decays [i-b]

\section{SVT tracking strategy}

The SVT separates the phases of pattern recognition and track fitting into two pipelined stages.
Pattern recognition is performed by the Associative Memory system, which identifies low resolution track candidates called roads [3i]. The roads found by the Associative Memory and the full resolution hits corresponding to them are passed to the Track Fitters which calculate track parameters. This is done using a linearized fitting algorithm implemented in hardware.

\subsection{Pattern recognition}

A track which traverses a multi-layer detector, produces a certain pattern of hits on each detector layer. Since detector resolution is finite, one could imagine to subdividing each detector layer into a finite number of elements with a size comparable to their resolution and to identify a track with the list of fired elements. Hit patterns corresponding to candidate tracks are stored in a memory, the Associative Memory, and are continuously compared in parallel to the data coming from the detector: a track candidate is found when all the hits corresponding to it are in the data. To reduce the size of the needed memory, pattern recognition is performed by the Associative Memory with a limited spatial resolution, for this purpose the Silicon Vertex Detector layers are segmented into $250 \mu \mathrm{m}$ wide superstrips, while the actual strip pitch is $\sim 60 \mu \mathrm{m}$.

The Associative Memory functions are implemented in a full custom VLSI chip with $0.7 \mu \mathrm{m}$ tech- 
nology $[\overline{4}|\overline{1}|,[\overline{5}]$. Each chip can store 128 patterns of 6 words (layers) of 12 bits. Operation of the chip has been tested up to $40 \mathrm{MHz}$, with the SVT specification being $30 \mathrm{MHz}$.

\subsection{Track fitting}

Track fitting is the problem of estimating the parameters of the candidate tracks found in the phase of pattern recognition by the Associative Memory. The SVT reconstructs tracks projected on the plane transverse to the beam axis and measures transverse momentum, azimuthal angle and impact parameter $\left(p_{t}, \phi, d\right)$. Track parameters are expressed as scalar products:

$$
p_{i}=\vec{f}_{i} \cdot \vec{x}+q_{i}
$$

where $p_{i}$ is one of the track parameters and $\vec{x}$ is the array containing hit positions and track curvature and azimuthal angle. The parameters $\vec{f}_{i}$ and $q_{i}$ are given by the linear expansion of the equations used to determine the track parameters. Within each $30^{\circ}$ SVX wedge $\vec{f}_{i}$ and $q_{i}$ are constants. Since variations of track parameters are small within a road, it is possible to expand $p_{i}$ around a position $x_{0}$ in the hit space (typically the lower road edge). The following algorithm can thus be used:

$$
\begin{aligned}
p_{i} & =\vec{f}_{i} \cdot\left(\vec{x}_{0}+\vec{d}\right)+q_{i} \\
p_{0 i}+\delta p_{i} & =\left(\vec{f}_{i} \cdot \vec{x}_{0}+q_{i}\right)+\vec{f}_{i} \cdot \vec{d}
\end{aligned}
$$

where $p_{0 i}=\vec{f}_{i} \cdot \vec{x}_{0}+q_{i}$ and $\delta p_{i}=\vec{f}_{i} \cdot \vec{d}$. The advantage of this algorithm is that the $p_{0 i}$ can be precalculated and stored in a look-up-table reducing the computational load required by eq. $\vec{d}$ varies within the road edges ( $\sim 250 \mu \mathrm{m}$ wide), a lower number of bits is necessary to have the full hit resolution.

SVT performance has been tested reconstructing real CDF run I data using a bit-level simulation program of the device and it has been proven that track parameters are measured with offline quality resolution: $\sigma_{p_{t}}=0.003 \cdot p_{t}^{2}, \sigma_{\phi}=1 \mathrm{mrad}$ and $\sigma_{d}=35 \mu \mathrm{m}$ (at $\left.p_{t}=2 \mathrm{GeV} / \mathrm{c}\right)$.

\section{All hadronic B decay trigger}

We have designed a trigger to select the $\mathrm{B}_{d}^{0} \rightarrow$ $\pi^{+} \pi^{-}$decay $[\underline{\bar{b}}]$ which is of fundamental importance in the study of $\mathrm{CP}$ violation in the SM.
The use of this trigger has been extended to similar decay channels like $\mathrm{B}_{s}^{0} \rightarrow D_{s}^{-} \pi^{+}$and $\mathrm{B}_{s}^{0} \rightarrow$ $D_{s}^{-} \pi^{+} \pi^{-} \pi^{+}$; the $\mathrm{D}_{s}^{-}$are reconstructed through the hadronic decays $\mathrm{D}_{s}^{-} \rightarrow \phi \pi^{-}$and $\mathrm{D}_{s}^{-} \rightarrow K^{* 0} K^{-}$ $[\overline{7}]$. This allows us to attack the problem of $\mathrm{B}_{s}^{0}$ mixing: since $\Delta m_{s}$ is expected to be large [i its measurement will require fully reconstructed $\mathrm{B}_{s}^{0}$ decays in order to achieve the necessary momentum resolution.

CDF has a three level trigger system with a maximum output of $50 \mathrm{kHz}$ at level 1 and $300 \mathrm{~Hz}$ at level 2. We have used a detailed simulation of the XFT and SVT processors and real CDF data from run I (1992-1996) to extrapolate the rates expected for the all hadronic $\mathrm{B}$ decay trigger in run II.

The trigger strategy is to select two oppositely charged tracks found by the XFT at level 1 . The XFT finds tracks with $p_{t}>1.5 \mathrm{GeV} / \mathrm{c}$ with a momentum resolution $\Delta p_{t} / p_{t}^{2}=0.015(\mathrm{GeV} / \mathrm{c})^{-1}$ and an azimuthal resolution of $\Delta \phi<1.5 \mathrm{mrad}$. To reduce the level 1 rate we have chosen to set a $p_{t}$ threshold of $2 \mathrm{GeV} / \mathrm{c}$ on both the selected tracks. We require $\Delta \phi<135^{\circ}$ to remove backto-back pairs from dijet events. The estimate for level 1 rate is $18 \mathrm{kHz}$ at the instantaneous luminosity $0.7 \cdot 10^{32} \mathrm{~cm}^{-2} \mathrm{sec}^{-1}$ expected at the beginning of run II.

At level 2 we use the SVT. The first step is to find pairs of tracks with significant $(>100 \mu \mathrm{m})$ impact parameters which also satisfy level 1 requirements. Next we require that the pair has a positive decay length. To optimize trigger efficiency on signal, we have developed separate selections for $\mathrm{B}_{d}^{0} \rightarrow \pi^{+} \pi^{-}$and $\mathrm{B}_{s}^{0}$ decays. We require $2^{\circ}<\Delta \phi<90^{\circ}$ for $\mathrm{B}_{s}^{0}$ decays and $20^{\circ}<$ $\Delta \phi<135^{\circ}$ for $\mathrm{B}_{d}^{0} \rightarrow \pi^{+} \pi^{-}$. Since in the case of $\mathrm{B}_{d}^{0} \rightarrow \pi^{+} \pi^{-}$the track pairs fully reconstruct the $\mathrm{B}_{d}^{0}$ candidate, which exits from the primary vertex, for $\mathrm{B}_{d}^{0} \rightarrow \pi^{+} \pi^{-}$we also require that the impact parameter of the two-track combination $d_{B}$ is consistent with zero. Our estimates also show that level 2 rates are well within the trigger bandwidth. As an example, in Figure $\prod_{11}^{\overline{1}}$ we show the level 2 trigger cross section dependence on the impact pararameter cut on both the selected tracks for the $\mathrm{B}_{d}^{0} \rightarrow \pi^{+} \pi^{-}$selection. In Figure $\overline{2}$ we show the efficiency of the level 2 trigger on the $\mathrm{B}_{d}^{0} \rightarrow \pi^{+} \pi^{-}$signal. Both the quoted level 2 
trigger cross section and signal efficiency include the level 1 cuts.

At level 3 the full event reconstruction will be available and we expect to keep the trigger rate at an easily managable level with full signal efficiency. Signal yields are estimated from Monte Carlo simulation. Using the value of the $\mathrm{B}_{d}^{0}$ production cross section measured by CDF [1 10 "], our efficiency estimate and assuming [i] $\left.\overline{1}_{1}\right]$ :

$$
B R\left(B_{d}^{0} \rightarrow \pi^{+} \pi^{-}\right)=\left(0.47_{-0.15}^{+0.18} \pm 0.13\right) \cdot 10^{-5}
$$

the expectation is to collect 7,000 events in $2 \mathrm{fb}^{-1}$. From similar estimates, assuming:

$$
\begin{gathered}
B R\left(B_{s}^{0} \rightarrow D_{s}^{-} \pi^{+}\right)=(0.30 \pm 0.04) \% \\
B R\left(B_{s}^{0} \rightarrow D_{s}^{-} \pi^{+} \pi^{-} \pi^{+}\right)=(0.80 \pm 0.25) \%
\end{gathered}
$$

we expect a total of $25,000 \mathrm{~B}_{s}^{0}$ events.

Work is in progress to evaluate the sensitivity to $\mathrm{CP}$ violation and mixing measurements. For the $\mathrm{B}_{d}^{0} \rightarrow \pi^{+} \pi^{-}$decay the question is whether it will be possible to extract the signal from the potentially enormous level of combinatorial background, while physics backgrounds such as $\mathrm{B}_{d}^{0} \rightarrow$ $K^{+} \pi^{-}$and $\mathrm{B}_{s}^{0} \rightarrow K^{+} K^{-}$can be extracted by making use of the invariant $\pi \pi$ mass distribution as well as the $\mathrm{dE} / \mathrm{dx}$ information provided by the COT and particle identification provided by the ToF. For the $\mathrm{B}_{s}^{0}$ channels the estimate is to have a Signal/Background between $1 / 2$ and $2 / 1$, with an estimated sensitivity on the mixing parameter $x_{s}$ up to $\sim 40$, which easily covers the currently favoured value of the SM for $x_{s}\left(18<x_{s}<27\right)$

\section{Conclusions}

In this paper we have reported on the new trigger strategies the CDF experiment is planning to adopt in run II to select fully hadronic B decays which are fundamental for measuring $\mathrm{CP}$ violation and $\mathrm{B}_{s}$ mixing. The trigger exploits the online precision tracking provided by the Silicon Vertex Tracker and selects track pairs with large impact parameters as candidates for hadronic B decays. Our studies show that the rates for this trigger are managable by the CDF data acquisition and that the efficiency on signals is sufficient to allow the collection of high statistics samples.

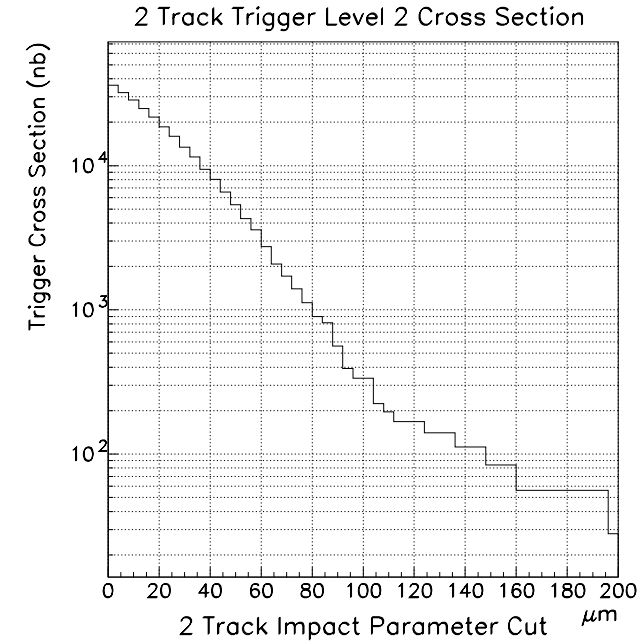

Figure 1: Level 2 cross section of the $\mathrm{B}_{d}^{0} \rightarrow \pi^{+} \pi^{-}$ selection as a function of the impact parameter cut.

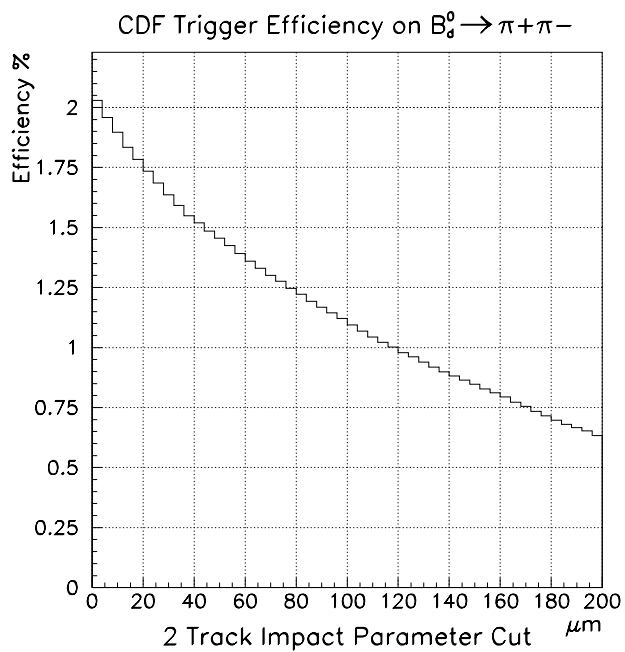

Figure 2: Level 2 trigger efficiency on the $\mathrm{B}_{d}^{0} \rightarrow$ $\pi^{+} \pi^{-}$signal as a function of the impact parameter cut.

\section{References}

[1] CDF II Collaboration, Technical Design Report, FERMILAB-Pub-96/390-E 1996.

[2] S. Belforte et al., The CDF trigger Silicon Vertex Tracker, IEEE Transactions on Nuclear Science, Vol 42, No. 4, August 1995.

W. Ashmanskas et al., The CDF Silicon Vertex Tracker: online precision tracking of the CDF Silicon Vertex Detector, to be published in the Proceedings of the 4th International Conference on Large Scale Applications and Radiation Hard- 
ness of Semiconductor Detectors, Firenze, Italy, June 23-25, 1999, FERMILAB-CONF-99/236-E.

[3] A. Bardi et al. A large Associative Memory system for the CDF Level 2 Trigger, to be published in the Proceedings of the 1998 IEEE Nuclear Science Symposium, November 8-14, 1998, Toronto, Canada.

[4] M. Dell'Orso et al., Nucl. Inst. and Meth. A 278 (1989), 436.

[5] S. R. Amendolia et al. The AM chip: a FullCustom CMOS VLSI Associative Memory for Pattern Recognition, IEEE Transactions on $\mathrm{Nu}-$ clear Science, Vol. 39, 1992.

[6] S. Donati, A strategy to measure the CP asymmetry in the $B_{d}^{0} \rightarrow \pi^{+} \pi^{-}$decay at $C D F, \mathrm{Ph} . \mathrm{D}$. Thesis, University of Pisa, November 1996.

G. Punzi, S. Donati and G. Gagliardi $A B_{d}^{0} \rightarrow$ $\pi^{+} \pi^{-}$trigger for $C D F$, Published Proceedings of the Workshop on B Physics at Hadron Accelerators, Snowmass, Colorado, 21 June - 2 July 1993, pp. $227-8$.

[7] The CDF II Collaboration, Proposal for Enhancement of the CDF II Detector: An Inner Silicon Layer and a Time of Flight Detector, Proposal P-909 submitted to the Fermilab Director and PAC, October 23, 1998.

The CDF II Collaboration, Update to Proposal P-909: Physics Performance of the CDF II with an Inner Silicon Layer and a Time-of-Flight Detector, submitted to the Fermilab Director and PAC, January 5, 1999.

[8] C. Caso et al. (Particle Data Group), Eur. Phys. J. C3, 1 (1998).

[9] S. Mele, IPhys. Rev. D $\mathbf{5 9}$ (1999) 113011.

[10] F. Abe et al., 'Phys. Rev.Lett. 75 (1995) 1451!.

[11] D. E. Jaffe, for the CLEO Collaboration, Rare $b$ decay results, these Proceedings. 